\title{
BMJ Open Behavioural and patient-individual determinants of quality of life, functioning and physical activity in older adults (MobilE-TRA 2): study protocol of an observational cohort study in a tertiary care setting
}

\author{
Benedict Katzenberger (10 ,1,2,3 Lars Schwettmann,, ${ }^{4,5}$ Martin Weigl, ${ }^{6}$ \\ Alexander Paulus, ${ }^{6}$ Sara Pedron, ${ }^{4,7}$ Sebastian Fuchs, ${ }^{1,3,6}$ Daniela Koller, ${ }^{1,2}$ \\ Eva Grill ${ }^{1,8}$
}

To cite: Katzenberger $B$, Schwettmann L, Weigl M, et al. Behavioural and patientindividual determinants of quality of life, functioning and physical activity in older adults (MobilE-TRA 2): study protocol of an observational cohort study in a tertiary care setting. BMJ Open 2021;11:e051915. doi:10.1136/ bmjopen-2021-051915

- Prepublication history for this paper is available online. To view these files, please visit the journal online (http://dx.doi org/10.1136/bmjopen-2021051915).

Received 31 March 2021 Accepted 12 November 2021

D) Check for updates

(C) Author(s) (or their employer(s)) 2021. Re-use permitted under CC BY-NC. No commercial re-use. See rights and permissions. Published by BMJ.

For numbered affiliations see end of article.

\section{Correspondence to}

Mr Benedict Katzenberger; benedict.katzenberger@med. uni-muenchen.de

\begin{abstract}
Introduction Vertigo, dizziness and balance problems (VDB) as well as osteoarthritis (OA) are among the health conditions with the greatest impact on mobility and social participation in older adults. Patients with VDB and OA were shown to benefit from specialised care such as vestibular rehabilitation therapy or joint replacement. However, these effects are not permanent and seem to disappear over time. One important reason might be a decreasing adherence to therapy recommendations. Findings from behavioural economics (BE) can help to shed light on individual effects on adherence behaviour and long-term outcomes of VDB and $O A$.
\end{abstract}

Objective Based on insights from BE concepts (ie, selfefficacy, intention, and time and risk preferences), MobilETRA 2 investigates the determinants of functioning and health-related quality of life (HRQLL) 3 and 12 months after discharge from total hip replacement (THR)/total knee replacement (TKR) in patients with $0 A$ and after interdisciplinary evaluation for VDB.

Methods and analysis MobilE-TRA 2 is a longitudinal observational study with data collection in two specialised tertiary care centres at the university hospital in Munich, Germany between 2020 and 2023. Patients aged 60 and older presenting for their first THR/TKR or interdisciplinary evaluation of VDB at Ludwig Maximilians University (LMU) hospital will be recruited for study participation. Three and twelve months after baseline assessment, all patients will receive a follow-up questionnaire. Mixed-effect regression models will be used to examine $B E$ concepts as determinants of adherence, HRQoL and functioning.

Ethics and dissemination The study was approved by the ethics committee at the medical faculty of the LMU Munich under the number 20-727. Results will be published in scientific, peer-reviewed journals and at national and international conferences. Findings will also be disseminated via newsletters, the project website and
Strengths and limitations of this study

- This observational prospective cohort study will provide comprehensive data of behavioural and patient-individual determinants of quality of life, functioning and physical activity in older adults at multiple time points.

- This study uses validated and well-established outcome measures.

- We investigate patients with vertigo, dizziness and balance problems or with osteoarthritis because these health conditions might have major impact on mobility and social participation in older adults.

- Data originate from one university hospital in Germany, so generalisability of the results is limited to this setting.

a regional conference for representatives of local and national authorities.

\section{INTRODUCTION}

Following economic modelling, adherence to therapy recommendations and other health behaviours, such as physical activity or a healthy diet, can be viewed as investments in one's own health. ${ }^{1}$ However, despite longterm advantages of these investments, individuals tend to deviate from these behaviours, even after educational efforts to provide information regarding their positive effects. Behavioural economics (BE) is a field of research that helps to explain such harmful deviations. ${ }^{2} 3$ Corresponding studies reveal that departures appear systematically and therefore can be predicted and explained using specific concepts, such as individual risk and time preferences. ${ }^{4}$ 
In the context of specialised hospital care, BE can help explaining patient-relevant outcomes after treatment, but also non-adherence to therapy recommendations. MobilE-TRA 2 uses insights from BE to shed light on individual effects on outcomes in patients with vertigo, dizziness and balance problems (VDB) as well as osteoarthritis (OA). These health conditions were chosen as they are among the health conditions with the greatest impact on mobility and social participation in older adults. ${ }^{5}$ The current project focuses on the investigation of the determinants of quality of life, functioning and physical activity, after a definite diagnosis has been made, and after therapy and rehabilitation have been initiated. Furthermore, adherence to recommendations may play a prominent role as a process variable, which could also be influenced by self-efficacy and risk or time preferences of patients.

With a prevalence of up to $50 \%$, VDB are frequent problems of patients aged 60 and older. The causes of VDB are often multifactorial. Distinct treatable vestibular disease entities, dizziness caused by medication, cardiovascular diseases or diabetes may align with symptoms of the ageing of vestibular, proprioceptive or somatosensory systems. By increasing postural instability, VDB are among the most apparent and prevalent causes for falls in aged adults. ${ }^{6}$ Furthermore, they significantly limit mobility and activities of daily life ${ }^{5}$ and restrict social participation. ${ }^{57}$ It is well established that aged adults with VDB benefit from vestibular rehabilitation therapy, regardless of the underlying pathology. However, it has been noted that old age is an independent predictor for unfavourable long-term outcomes, even after evidence-based and interdisciplinary treatment. ${ }^{8}$

With respect to VDB, only a limited number of $\mathrm{BE}$ concepts have been investigated to date. Patients showing resilience and high self-efficacy were less likely to develop secondary, chronic forms of functional vertigo. ${ }^{9}$ Likewise, persons with high general self-efficacy reported more confronting, active behaviour to address their visual height intolerance. ${ }^{10}$

Likewise, $\mathrm{OA}$ is a major cause of mobility restrictions in older adults. ${ }^{11}$ Persons with OA have an increased risk for cardiovascular and dementia associated mortality, with low levels of physical activity contributing to this excess mortality. ${ }^{12}$ Several evidence-based recommendations and guidelines are available for the management of OA. ${ }^{13}$ Specifically, after joint replacement, physical therapy, home exercises and increased physical activity are effective methods to reduce limitations of patients' functioning and mobility. ${ }^{14}$ However, these effects are not permanent and seem to disappear over time. One major aspect contributing to this decline is non-adherence to physical activity recommendations, ${ }^{16}$ which concerns up to $50 \%$ of OA patients and might be one decisive factor jeopardising positive surgery outcomes. ${ }^{17}$ This may lead to reduced mobility and low physical functioning levels after joint replacement and increase the likelihood for subsequent revision. ${ }^{18}$ Besides non-adherence to physical activity recommendations, there might be several other factors, which play a role in determining long-term outcomes after major treatment decisions, for example, after total hip or knee replacement (THR/TKR), but their role has yet to be acknowledged and understood.

In general, attitudes and motivation of patients are among the determinants for therapeutic success after THR/TKR. ${ }^{19}{ }^{20}$ Physical, social and socioeconomic factors, but also psychological aspects including selfefficacy, intentions, or expectancies for the future, serve as potential barriers to better outcomes. ${ }^{21-25}$ Regarding $\mathrm{BE}$, stronger orientation to the present appears to be associated with less healthy behaviour. ${ }^{4627}$ However, it is unclear if increased risk tolerance promotes or inhibits adherence to recommended exercise. ${ }^{47}$

In summary, too little attention has been paid to the role of $\mathrm{BE}$ concepts for long-term outcomes of $\mathrm{OA}$ and VDB. In fact, these factors may need to be considered not only in the preoperative phase, but also during discharge and rehabilitation planning and aftercare. Corresponding knowledge can be used to refine care pathways and to inform healthcare practice, potentially identifying persons at higher risk for adverse outcomes and tailoring specific interventions to improve adherence.

MobilE-TRA 2 is part of the second phase of MobilE-Net (Enabling participation by enabling MOBILity in older adults-Evidence-based health care research Network). MobilE-Net 2 is composed of three projects, which aim to develop multiprofessional care pathways targeted at older adults to reduce the burden of disability and to promote healthy ageing, mobility and participation. Based on insights from $\mathrm{BE}$, the second phase of the subproject MobilE-TRA 2 investigates the determinants of functioning and HRQoL for patients with OA at 3 and 12 months after discharge from THR/TKR and for patients with VDB at 3 and 12 months after interdisciplinary evidence-based evaluation. In addition, adherence to recommendations will be considered as a major process variable. Results are of particular importance for the other projects of MobilE-Net to explain unwanted variations in outcomes of an implementation process. We expect to deliver insights that can directly be applied in ambulatory care and in discharge and rehabilitation planning.

Specifically, we want

1. to examine BE concepts as determinants of adherence,

2. to examine $\mathrm{BE}$ concepts as determinants of HRQoL and functioning, and

3. to investigate the moderation effect of BE concepts on associations between physical activity and individual factors such as body mass index (BMI) or pain.

\section{METHODS AND ANALYSIS}

\section{Study design}

MobilE-TRA 2 is a prospective observational cohort study among patients with OA after THR/TKR and patients with VDB after interdisciplinary evidence-based evaluation 
in two specialised tertiary care centres at the university hospital in Munich, Germany. The patient recruitment will take place from November 2020 until March 2022 and the data collection will end in March 2023 with the last patient completing the twelve months follow-up. The longitudinal design of the study captures changes in relevant outcomes. The study is monocentric to avoid variations in case ascertainment as well as surgical and medical treatment standards.

\section{Participants and recruitment}

Patient recruitment for MobilE-TRA 2 will take place at the Department of Orthopaedics, Physical Medicine and Rehabilitation (OPMR) and the German Centre for Vertigo and Balance Disorders (DSGZ) at the Ludwig Maximilians University (LMU) hospital in Munich. About 300 patients per year are admitted for first THR/ TKR surgery at the Department OPMR. The DSGZ treats over 3000 ambulatory patients per year. Patients at OPMR and DSGZ obtain a rigorous clinical workup in line with current guidelines. ${ }^{1328}$ Typically, in the German system, patients after THR/TKR will undergo 3 weeks of postacute inpatient musculoskeletal rehabilitation and will be discharged from rehabilitation with a recommendation for physical therapy and physical activity. Patients with VDB will receive a recommendation for further measures in line with the recommendations of the Bárány Society ${ }^{29}$ to be initiated by their primary care physician, potentially including physical therapy, physical activity or medication.

Patients aged 60 and older presenting at the participating centres for first THR/TKR or first interdisciplinary evaluation of VDB at the LMU hospital will be recruited for study participation. Patients with terminal disease, cognitive impairment, insufficient command of the German language or physical problems preventing participation, such as sensory impairments (bad sight or hearing) or a very low energy level, will be excluded. Patients will be recruited from both departments during their first consultations, that is, prior to surgery for patients with OA and during the first visit at the DSGZ for patients with VDB. Additionally, OA inpatients are recruited at the hospital 1 day ahead of their surgery. The recruitment is performed by trained members of the study team.

Patients participating in the MobilE-TRA 2 study will receive the patient information, the informed consent documents and the baseline questionnaire from the tertiary care centre. The patients in the study do not receive an incentive for study participation. In case of consent, they fill out these forms and hand them back to the study centre. Patients can also choose to complete the questionnaire at home and send it back to the respective study centre. The data collection in this study will be done with the help of pseudonymised identification keys during study conduct. For further information, refer to the section ethics and dissemination of this study protocol.
For the follow-up surveys after 3 and 12 months, a cover letter with instructions and the questionnaires will be sent via postal mail from the study teams to the patients' addresses. If no questionnaire is sent back within a month after the original follow-up invitation, one reminder will be sent. If the participant does not respond to the reminder, the data will be considered missing for the first follow-up. In the second follow-up, 1 month after an unanswered reminder, a non-responder questionnaire will be provided to explore the reasons for withdrawal and to obtain a crude estimate on health status based on a small set of questions.

The recruitment of the study is taking place during the SARS-CoV-2 pandemic. This emergency forced hospitals to take measures to prevent a patient overload and to grant back-up capacities for patients in need of intensive care, for example, by cancelling all routine operations that are not immediately indispensable. This may lead to a potential preselection of the study participants based on their constitution and the severity of the underlying disease. These challenges will be addressed by a careful documentation of the recruitment process and the specifications in force.

\section{Patient and public involvement}

Before starting the actual data collection, the questionnaires were pretested on a total of 11 patients at both the OPMR and the DSGZ at the LMU hospital in Munich from October to November 2020, using a think-out-loud approach. Patients did not indicate any major problems, such as ambiguous instructions, when filling out the questionnaire. The results of this pretest then were then used to revise and finalise the questionnaires. Results of the main study will be disseminated to study participants via the project website.

\section{Measures}

The primary outcomes of the study are generic quality of life, OA-specific physical functioning in patients with $\mathrm{OA}$ and vertigo-specific physical and psychosocial functioning in patients with VDB after 3 and 12 months, measured using validated instruments. Generic quality of life is measured through the EuroQol Five-Dimensional Five-Level Questionnaire (EQ-5D-5L) of the EuroQol Group. ${ }^{30}$ For summary valuation, patients' report on the visual analogue scale and the German value set for utilities based on EQ-5D-5 $\mathrm{L}^{31}$ will be used.

OA-specific physical functioning in persons with hip and/or knee OA is assessed using the German version of the Western Ontario and McMaster Universities Osteoarthritis Index (WOMAC).$^{32} 33$ The WOMAC is an OA-specific tridimensional patient relevant outcome measure with 24 questions ( 5 on pain, 2 on stiffness and 17 on physical functioning). The main focus of the WOMAC is on the domain mobility that is addressed by 15 of the 24 questions. ${ }^{34}$ This study applies the Likert scaled format with 5 response categories $(0=$ none, $1=$ mild, $2=$ moderate, $3=$ severe, $4=$ extreme). The sum of the assigned values 
results in the score of each scale. The WOMAC has been proven valid, reliable and responsive ${ }^{32}$ and is arguably the most frequently used OA-specific patient relevant outcome measure.

Patients with VDB will be asked to fill out the German version of Dizziness Handicap Inventory (DHI) ${ }^{35}$ which assesses disability caused by dizziness and unsteadiness on everyday activities, including activity limitation, participation restrictions, and experienced difficulties.

Adherence behaviour to therapy recommendations, physical activity, pain and satisfaction is used as secondary outcome measure. Adherence behaviour to therapy recommendations is addressed with three items dealing with adherence to disease-specific exercises and physical activity (sporting activities and general regular exercise) ${ }^{16}$ and one item dealing with weight control. OA specific pain is assessed using the pain scale of the WOMAC. ${ }^{36}$ Pain and satisfaction in patients with $\mathrm{OA}$ were further assessed by single-item measures following Rolfson et al (2016) ${ }^{37}$ The pain question asks: 'During the past 4 weeks, how would you describe the pain you usually have in your affected hip/knee?'. Response options are none, very mild, mild, moderate and severe. The satisfaction question asks: 'How satisfied are you with the results of your joint replacement that you received 3 months (12 months) ago?', with the response options very dissatisfied, dissatisfied, neutral, satisfied and very satisfied.

Physical activity for patients with OA or VDB is measured using the self-administered Global Physical Activity Questionnaire (GPAQ) ${ }^{38}{ }^{39}$ The WHO developed the GPAQ for physical activity surveillance. Fifteen questions collect information in the three domains activity at work, travel to and from places, and recreational activities. In addition, one single question asks for sedentary behaviour. The weekly energy expenditure in Metabolic Equivalents is estimated using the frequencies and durations of moderate and vigorous physical activity in each domain in a usual week. The GPAQ is validated in Germany and many other countries ${ }^{40}$ and has been applied to various populations including patients with OA. ${ }^{41}$

Concepts of BE are used as exposure. Risk attitudes are measured via two questions including an 11-point Likert scale, where individuals can state their attitude to risk in general and in the health domain from (0) 'not at all ready to take risks' to (10) 'very likely to take risks'. ${ }^{42}$ Selfefficacy is rated using the validated Allgemeine Selbstwirksamkeit Kurzskala questionnaire, where individuals are asked to answer three questions on general selfefficacy. ${ }^{43}$ Additionally, a further question focuses on selfefficacy concerning physical activity supplemented by an item addressing intention to carry out physical activities. ${ }^{44}$

Time preferences are measured by using three distinct instruments. First, a questionnaire instrument with six items is applied creating a series of binary choices between hypothetical amounts of money now or in the closer future and alternative amounts in the further future. From these choices, individual discount rates can be derived. ${ }^{45}$ Second, a qualitative item based on Borghans and Golsteyn (2006) ${ }^{46}$ asks respondents to indicate their present-future tradeoff on a 5-point Likert scale. Third, respondents' self-assessed willingness to wait has to be stated on an 11-point Likert scale. ${ }^{47}$

In order to consider potential confounding, information on age, sex, education, occupation, marital status, living conditions, alcohol consumption and smoking behaviour is collected in the questionnaire. We further gathered information about comorbidities of the heart, lung, liver and kidneys as well as neurological diseases, high blood pressure, inflammatory joint diseases and further diseases specifically indicated by the participants. Depression will be assessed using the Patient Health Questionnaire. ${ }^{48}$ The exact diagnosis of VDB will be obtained from the patient documentation at the DSGZ. In patients with OA, weight and height are measured routinely as a part of the premedical examination prior to the surgery at the OPMR and are used to calculate the BMI.

German translations were not available for the concepts of $\mathrm{BE}$, the pain and satisfaction in patients with $\mathrm{OA}$, and the adherence behaviour to therapy recommendations. These items thus were independently translated by at least two members of the study team. Discrepancies between the translations were discussed and resolved between the translators. The translated versions then were examined by the respective expert within the study team for each item in order to ensure that the translation did not change the construct of interest.

An overview of research outcomes and instruments used, including the number of items and corresponding references, is shown in table 1 .

\section{Sample size}

Sample size calculation for THR/TKR is based on a minimally important difference (MID) of 8 for TKR and 9 for THR and a SD of 18 of the EQ-5D-5L. ${ }^{49}$ Thus, 37 THR and 47 TKR patients are needed to estimate the mean with a power of 0.8 (alpha=0.05). Assuming a loss to follow-up of $20 \%$ between baseline and follow-ups, we need to include $131(58+73)$ patients at baseline. Attrition rate is reasonable, based on our previous experiences with primary care patients where we found that $88 \%$ of patients of this age group had responded to the first postal follow-up. MID for the EQ-5D-5L are not available for VDB, so based on previous work, ${ }^{50}$ we assume 10.0 points on the $\mathrm{DHI}^{35}$ as a clinically relevant difference for patients with VDB. Assuming an SD of 25, a sample size of 52 will allow estimating the mean with a power of 0.8 (alpha $=0.05)$. Assuming a loss to follow-up of $20 \%$, we need to include 81 patients at baseline. For patients with VDB, different underlying pathologies will have to be considered. Thus, we plan to sample 81 patients from each of the four main somatic aetiologies of VDB, namely Benign Paroxysmal Positional Vertigo, Menière's disease, vestibular migraine, and functional vertigo, yielding a target sample size of 324. 
Table 1 Research outcomes and instruments used, including the number of items

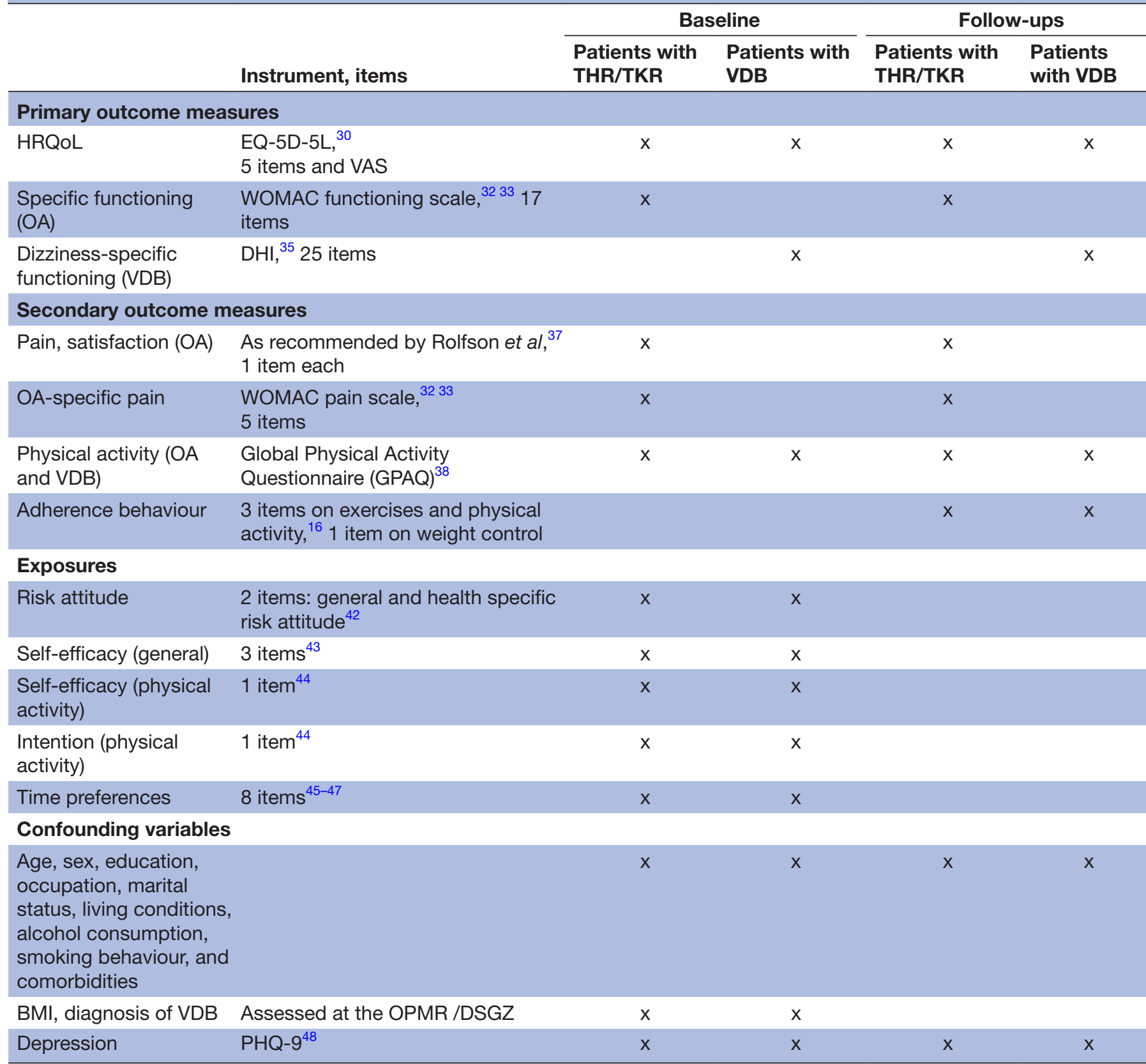

BMI, body mass index; DHI, Dizziness Handicap Inventory; DSGZ, German Centre for Vertigo and Balance Disorders; EQ-5D-5L, EuroQol Five-Dimensional Five-Level Questionnaire; HRQoL, health-related quality of life; OA, osteoarthritis; OPMR, Department of Orthopaedics, Physical Medicine and Rehabilitation; THR/TKR, total hip or knee replacement; VAS, visual analogue scale; VDB, vertigo, dizziness and balance problems; WOMAC, Western Ontario and McMaster Universities Osteoarthritis Index.

\section{Data analysis}

In a first step, BE concepts, HRQoL and functioning will be analysed descriptively. Differences will be compared using $\chi^{2}$ tests for categorical variables and analysis of variance for metric variables, which are $\log$ transformed if necessary.

Second, mixed-effect regression models will be used in the sample with patients with OA and VDB to examine $\mathrm{BE}$ concepts as determinants of adherence, HRQoL and functioning. This method is appropriate to investigate longitudinal data with more than one wave of data. ${ }^{51}$
Third, to investigate the impact of BE and psychological concepts as confounders for the association between physical activity and other factors, we will conduct stratified analyses and incorporate interaction terms in the regression models.

\section{ETHICS AND DISSEMINATION}

The study was approved by the ethics committee at the medical faculty of the Ludwig Maximilian University Munich under the number 20-727. Patients are supplied 
with sufficient information about their participation in the study and are only included if written informed consent is present. Participants will not be exposed to any medical risk due to study participation. The study is conducted in accordance with the Declaration of Helsinki. Data collection in this study will be done with the help of pseudonymised identification keys during study conduct. All links between the pseudonymised keys and person identifying information such as name or address will be destroyed after study completion. Conclusion about the actual patient will then no longer be possible and data will be de facto anonymised. Data will be double pseudonymised before being forwarded for data analysis to project partners only.

Results will be published in scientific, peer-reviewed journals and at national and international conferences. Results will be disseminated via newsletters, the project website and a regional conference for representatives of local and national authorities. The data set will be available for project researchers on request.

\section{Author affiliations}

${ }^{1}$ Institute for Medical Information Processing, Biometry and Epidemiology, Ludwig Maximilians University Munich, Munich, Germany

${ }^{2}$ Munich Center of Health Sciences, Ludwig Maximilians University Munich, Munich, Germany

${ }^{3}$ Pettenkofer School of Public Health, Ludwig Maximilians University Munich, Munich, Germany

${ }^{4}$ Institute of Health Economics and Health Care Management, Helmholtz Center Munich German Research Center for Environmental Health, Neuherberg, Germany

${ }^{5}$ Department of Economics, Martin Luther University Halle Wittenberg, Halle, Germany

${ }^{6}$ Department of Orthopaedics and Trauma Surgery, Musculoskeletal University Center Munich (MUM), University Hospital Munich, Munich, Germany

${ }^{7}$ Professorship of Public Health and Prevention, Faculty of Sport and Health Science, Technical University of Munich, Munich, Germany

${ }^{8}$ German Centre for Vertigo and Balance Disorders, University Hospital Munich, Munich, Germany

Acknowledgements The authors acknowledge the patients' willingness to participate, their appreciated support and their valuable feedback in the pilot testing of the questionnaire. The authors thank Michael Klein and Karoline Bögle for their valuable contribution to the data collection of this study.

Contributors EG, LS, MW and AP are the principal investigators. They conceived and supervised the project. BK coordinated the study. EG, LS, MW, AP and DK contributed to the conception of the cohort and design of the project. DK, BK and EG are responsible for the study's quality assessment. SF is responsible for the data management at the OPMR and preliminary analysis of the osteoarthritis data. LS and SP are responsible for secondary data management, preliminary analysis and preparation and contribute expertise on behavioural economics. BK, EG, LS, MW SP, $\mathrm{SF}$ and AP drafted the manuscript. All authors approved and critically revised the final manuscript.

Funding This work is part of the project "Munich Network Healthcare ResearchMobilE-Net" and was supported by the German Federal Ministry of Education and Research grant number 01GY1913A.

\section{Competing interests None declared.}

Patient and public involvement Patients and/or the public were involved in the design, or conduct, or reporting, or dissemination plans of this research. Refer to the Methods section for further details.

Patient consent for publication Not applicable.

Ethics approval The study was approved by the ethics committee at the medical faculty of the Ludwig Maximilian University Munich under the number 20--727.

Provenance and peer review Not commissioned; externally peer reviewed.
Open access This is an open access article distributed in accordance with the Creative Commons Attribution Non Commercial (CC BY-NC 4.0) license, which permits others to distribute, remix, adapt, build upon this work non-commercially, and license their derivative works on different terms, provided the original work is properly cited, appropriate credit is given, any changes made indicated, and the use is non-commercial. See: http://creativecommons.org/licenses/by-nc/4.0/.

ORCID iD

Benedict Katzenberger http://orcid.org/0000-0003-1043-4104

\section{REFERENCES}

1 Grossman M. On the concept of health capital and the demand for health. J Polit Econ 1972:80:223-55

2 Leonard TC, Thaler RH, Cass R. Sunstein, Nudge: improving decisions about health, wealth, and happiness. Springer, 2008.

3 Ariely D, Jones S. Predictably irrational. NY: Harper Audio New York, 2008.

4 van der Pol M, Hennessy D, Manns B. The role of time and risk preferences in adherence to physician advice on health behavior change. Eur J Health Econ 2017;18:373-86.

5 Mueller M, Strobl R, Jahn K, et al. Impact of vertigo and dizziness on self-perceived participation and autonomy in older adults: results from the KORA-Age study. Qual Life Res 2014;23:2301-8.

6 Agrawal Y, Carey JP, Della Santina CC, et al. Disorders of balance and vestibular function in US adults: data from the National health and nutrition examination survey, 2001-2004. Arch Intern Med 2009;169:938-44.

7 Bronstein AM, Golding JF, Gresty MA, et al. The social impact of dizziness in London and Siena. J Neurol 2010;257:183-90.

8 Obermann M, Bock E, Sabev N, et al. Long-term outcome of vertigo and dizziness associated disorders following treatment in specialized tertiary care: the dizziness and vertigo registry (diver) study. J Neurol 2015;262:2083-91

9 Tschan R, Best C, Beutel ME, et al. Patients' psychological wellbeing and resilient coping protect from secondary somatoform vertigo and dizziness (SVD) 1 year after vestibular disease. J Neurol 2011;258:104-12.

10 Grill E, Schäffler F, Huppert D, et al. Self-Efficacy beliefs are associated with visual height intolerance: a cross-sectional survey. PLoS One 2014:9:e116220.

11 Ettinger WH, Afable RF. Physical disability from knee osteoarthritis: the role of exercise as an intervention. Med Sci Sports Exerc 1994;26:1435-40.

12 Nüesch E, Dieppe P, Reichenbach S, et al. All cause and disease specific mortality in patients with knee or hip osteoarthritis: population based cohort study. BMJ 2011;342:d1165.

13 Nelson AE, Allen KD, Golightly YM, et al. A systematic review of recommendations and guidelines for the management of osteoarthritis: the chronic osteoarthritis management initiative of the U.S. bone and joint initiative. Semin Arthritis Rheum 2014;43:701-12.

14 Jette DU, Hunter SJ, Burkett L, et al. Physical therapist management of total knee arthroplasty. Phys Ther 2020;100:1603-31.

15 Lenguerrand E, Artz N, Marques E, et al. Effect of Group-Based outpatient physical therapy on function after total knee replacement: results from a multicenter randomized controlled trial. Arthritis Care Res 2020;72:768-77.

16 Pisters MF, Veenhof C, Schellevis FG, et al. Exercise adherence improving long-term patient outcome in patients with osteoarthritis of the hip and/or knee. Arthritis Care Res 2010;62:1087-94.

17 Argent R, Daly A, Caulfield B. Patient involvement with home-based exercise programs: can connected health interventions influence adherence? JMIR Mhealth Uhealth 2018;6:e47

18 Devane P, Horne G, Gehling DJ. Oxford hip scores at 6 months and 5 years are associated with total hip revision within the subsequent 2 years. Clin Orthop Relat Res 2013;471:3870-4.

19 Hurley M, Dickson K, Hallett R, et al. Exercise interventions and patient beliefs for people with hip, knee or hip and knee osteoarthritis: a mixed methods review. Cochrane Database Syst Rev 2018;4:CD010842.

20 Mistry JB, Elmallah RDK, Bhave A, et al. Rehabilitative guidelines after total knee arthroplasty: a review. J Knee Surg 2016;29:201-17.

21 Essery R, Geraghty AWA, Kirby S, et al. Predictors of adherence to home-based physical therapies: a systematic review. Disabil Rehabil 2017;39:519-34.

22 Hodges A, Harmer AR, Dennis S, et al. Prevalence and determinants of physical activity and sedentary behaviour before and up to 12 months after total knee replacement: a longitudinal cohort study. Clin Rehabil 2018;32:1271-83. 
23 Papalia R, Vasta S, Tecame A, et al. Home-based vs supervised rehabilitation programs following knee surgery: a systematic review. Br Med Bull 2013;108:55-72.

24 Engel C, Hamilton NA, Potter PT, et al. Impact of two types of expectancy on recovery from total knee replacement surgery (TKR) in adults with osteoarthritis. Behav Med 2004;30:113-23.

25 Magklara E, Burton CR, Morrison V. Does self-efficacy influence recovery and well-being in osteoarthritis patients undergoing joint replacement? A systematic review. Clin Rehabil 2014;28:835-46.

26 Kosteas VD. Physical activity and time preference. Int $J$ Health Econ Manag 2015;15:361-86.

27 Leonard T, Shuval K, de Oliveira A, et al. Health behavior and behavioral economics: economic preferences and physical activity stages of change in a low-income African-American community. Am $J$ Health Promot 2013;27:211-21.

28 DGfOuUeV. Evidenz- und konsensbasierte Indikationskriterien zur Hüfttotalendoprothese bei Coxarthrose: AWMF online 2021.

29 Bisdorff A, Von Brevern M, Lempert T, et al. Classification of vestibular symptoms: towards an international classification of vestibular disorders. J Vestib Res 2009;19:1-13.

30 Herdman M, Gudex C, Lloyd A, et al. Development and preliminary testing of the new five-level version of EQ-5D (EQ-5D-5L). Qual Life Res 2011;20:1727-36.

31 Ludwig K, Graf von der Schulenburg J-M, Greiner W. German value set for the EQ-5D-5L. Pharmacoeconomics 2018;36:663-74.

32 Bellamy N, Buchanan WW, Goldsmith $\mathrm{CH}$, et al. Validation study of WOMAC: a health status instrument for measuring clinically important patient relevant outcomes to antirheumatic drug therapy in patients with osteoarthritis of the hip or knee. J Rheumatol 1988;15:1833-40.

33 Stucki G, Meier D, Stucki S, et al. [Evaluation of a German version of WOMAC (Western Ontario and McMaster Universities) Arthrosis Index]. Z Rheumatol 1996;55:40-9.

34 Weigl M, Cieza A, Harder M, et al. Linking osteoarthritis-specific health-status measures to the International classification of functioning, disability, and health (ICF). Osteoarthritis Cartilage 2003;11:519-23.

35 Kurre A, van Gool CJAW, Bastiaenen CHG, et al. Translation, cross-cultural adaptation and reliability of the German version of the dizziness handicap inventory. Otol Neurotol 2009;30:359-67.

36 Bellamy N. The WOMAC knee and hip osteoarthritis indices: development, validation, globalization and influence on the development of the AUSCAN hand osteoarthritis indices. Clin Exp Rheumatol 2005;23:S148-53.

37 Rolfson O, Bohm E, Franklin P, et al. Patient-Reported outcome measures in arthroplasty registries report of the patient-reported outcome measures Working group of the International Society of arthroplasty registries Part II. recommendations for selection, administration, and analysis. Acta Orthop 2016;87(Suppl 1):9-23.

38 Armstrong T, Bull F. Development of the world Health organization global physical activity questionnaire (GPAQ). J Public Health 2006;14:66-70.

39 Chu AHY, Ng SHX, Koh D, et al. Reliability and validity of the selfand Interviewer-Administered versions of the global physical activity questionnaire (GPAQ). PLoS One 2015;10:e0136944.

40 Wanner M, Hartmann C, Pestoni G, et al. Validation of the global physical activity questionnaire for self-administration in a European context. BMJ Open Sport Exerc Med 2017;3:e000206.

41 Wanaratna K, Muangpaisan W, Kuptniratsaikul V, et al. Prevalence and factors associated with frailty and cognitive frailty among community-dwelling elderly with knee osteoarthritis. J Community Health 2019;44:587-95.

42 Dohmen T, Falk A, Huffman D, et al. Individual risk attitudes: measurement, determinants, and behavioral consequences. J Eur Econ Assoc 2011;9:522-50.

43 Beierlein C, Kemper C, Kovaleva A. Kurzskala zur Erfassung allgemeiner Selbstwirksamkeitserwartungen (ASKU). Methoden, Daten, Analysen 2013;7:251-78.

44 Hall PA, Fong GT, Cheng AY. Time perspective and weight management behaviors in newly diagnosed type 2 diabetes: a mediational analysis. J Behav Med 2012;35:569-80.

45 van der Pol M. Health, education and time preference. Health Econ 2011;20:917-29.

46 Borghans L, Golsteyn BHH. Time discounting and the body mass index. Evidence from the Netherlands. Econ Hum Biol 2006;4:39-61.

47 Falk A, Becker A, Dohmen T, et al. Global evidence on economic preferences. Q J Econ 2018;133:1645-92.

48 Kroenke K, Spitzer RL, Williams JB. The PHQ-9: validity of a brief depression severity measure. J Gen Intern Med 2001;16:606-13.

49 Conner-Spady BL, Marshall DA, Bohm E, et al. Comparing the validity and responsiveness of the EQ-5D-5L to the Oxford hip and knee scores and SF-12 in osteoarthritis patients 1 year following total joint replacement. Qual Life Res 2018;27:1311-22.

50 Grill E, Penger M, Kentala E. Health care utilization, prognosis and outcomes of vestibular disease in primary care settings: systematic review. J Neurol 2016;263(Suppl 1):36-44.

51 Singer JD, Willett JB, Willett JB. Applied longitudinal data analysis: modeling change and event occurrence. Oxford university press, 2003. 